\title{
Dynamical phase transition in one-dimensional kinetic Ising model with nonuniform coupling constants
}

\author{
Mohammad Khorrami1 \& Amir Aghamohammad² \\ Department of Physics, Alzahra University, Tehran 19384, IRAN
}

PACS numbers: 64.60.-i, 05.40.-a, 02.50.Ga

Keywords: reaction-diffusion, phase transition, Glauber model

\begin{abstract}
An extension of the Kinetic Ising model with nonuniform coupling constants on a one-dimensional lattice with boundaries is investigated, and the relaxation of such a system towards its equilibrium is studied. Using a transfer matrix method, it is shown that there are cases where the system exhibits a dynamical phase transition. There may be two phases, the fast phase and the slow phase. For some region of the parameter space, the relaxation time is independent of the reaction rates at the boundaries. Changing continuously the reaction rates at the boundaries, however, there is a point where the relaxation times begins changing, as a continuous (nonconstant) function of the reaction rates at the boundaries, so that at this point there is a jump in the derivative of the relaxation time with respect to the reaction rates at the boundaries.
\end{abstract}

\footnotetext{
${ }^{1}$ e-mail: mamwad@mailaps.org

e-mail: mohamadi@alzahra.ac.ir
} 


\section{Introduction}

The Glauber dynamics was originally proposed to study the relaxation of the Ising model towards equilibrium states [1. It is a simple non-equilibrium model of interacting spins with spin-flip dynamics. Kinetic models based on the Ising model, for example the Glauber model or the Kawasaki spin-pair exchange mechanism model 2, are phenomenological models and have been extensively studied 1-6. It has been shown that there is a relationship between the onedimensional kinetic Ising model at zero temperature and diffusion annihilation in one dimension [3]. In [4], using a damage spreading method, the sensitivity of the time evolution of a kinetic Ising model with Glauber dynamics against the initial conditions has been investigated. The full time dependence of the spacedependent magnetization and of the equal time spin-spin correlation functions were studied in [5]. Non-equilibrium two-time correlation and response functions for the ferromagnetic Ising chain with Glauber dynamics have been studied in [6.,7].

Combinations of the Glauber and the Kawasaki dynamics have been also considered 8-10. Most studies are focused on uniform lattices where reaction rates are site-independent. It is known that the ordinary Glauber model on a one-dimensional lattice with boundaries at any temperature, shows a dynamical phase transition [11. The dynamical phase transition is controlled by the rate of spin flip at the boundaries, and is a discontinuous change of the derivative of the relaxation time towards the stationary configuration. Among the simplest generalizations beyond a uniform system is a lattice with alternating rates. In [12 14, the steady state configurational probabilities of an Ising spin chain driven out of equilibrium by a coupling to two heat baths have been investigated. An example is a one-dimensional Ising model on a ring, in which the evolution is according to a generalization of Glauber rates, such that spins at even (odd) lattice sites experience a temperature $T_{e}\left(T_{o}\right)$. In this model the detailed balance is violated. The response function to an infinitesimal magnetic field for the Ising-Glauber model with arbitrary exchange couplings has been studied in [15. Other generalizations of the Glauber model consist of, for example, alternating-isotopic chains and alternating-bound chains ( [16] for example). In [17, an asymmetric generalization of the zero-temperature Glauber model on a lattice with boundaries was introduced. There it was shown that in the thermodynamic limit (when the lattice becomes infinite) the system shows two kinds of phase transitions. One of these is a static phase transition, the other a dynamic one. The static phase transition is controlled by the bulk reaction rates, and is a discontinuous change of the behavior of the derivative of the stationary magnetization at the end points, with respect to the reaction rates. The dynamic phase transition is controlled by the spin flip rates of the particles

at the end points, and is a discontinuous change of the relaxation time towards the stationary configuration. Other phase transitions induced by boundary conditions have also been studied ( [18 20, for example). Another generalization of the Glauber model was introduced in [21. In this generalization, the processes are the same as those of the ordinary Glauber model, but the rates depend on 
three free parameters, rather than one free parameter as in the ordinary Glauber model. Finally, this model was further generalized to the case where the number of interacting sites is more than three and the number of states at each site is more than two. This model too violates detailed balance. In [22, an inherent spin anisotropy (kinetic disorder) in the Glauber- Ising model was introduced. It was shown both analytically and numerically, that there is a slow logarithmic factor in the decay of the density of kinks at large times. In [23], the problem of the effect of quenched impurities on one-dimensional non-equilibrium GlauberIsing-type models has been investigated numerically. It was shown that the model has a continuous phase transition to an absorbing state. Also a meanfield approach has been used to study the Glauber-type stochastic dynamics of a model on a square lattice in which two interpenetrating square sublattices have spins that can take two values, alternated with spins that can take the four values 24 .

The behavior of an Ising model with nonuniform coupling constants at low temperatures on a one dimensional periodic lattice has been discussed in 25]. The static behavior of an Ising model with nonuniform coupling constants on a one-dimensional lattice with boundaries was investigated in [26]. Detailed balance was used in [25,26] to propose reaction rates for the system. In [26] the time-independent solution to the evolution equation of the expectation values of spins was studied. This solution was expressed in terms of a transfer matrix. While it is true that the ordinary Ising model does not exhibit any phase transition in finite temperatures (the expectation values of the spins vanish if there is no external magnetic field), this is not necessarily the case for the Ising model with inhomogeneous boundary conditions. It was shown that in the thermodynamic limit different phases could occur for this system, according to whether the eigenvalues of the transfer matrix are less than or larger than one.

In this paper the dynamical properties of kinetic Ising model with nonuniform coupling constants are investigated. The nonuniformity could arise from either a nonuniformity in the coupling constants or a nonuniformity in the temperature. However, as long as the dynamics of the system is considered, only the ratios of the coupling constants and the temperature are important, so without loss of generality one can assume the temperature to be constant and put all the nonuniformity in the coupling constant. So a nonuniformity in the (effective) coupling constants could be due to a nonuniformity in the temperature. It could also be due to a nonuniformity in the interaction between neighboring spins, or due to a nonuniformity in some sort of inertia, which opposes the changes in the state of the system.

It is shown that there are cases where the system exhibits a dynamical phase transition. There are two phases: the fast phase and the slow phase. For some region of the parameter space, the relaxation time is independent of the reaction rates at the boundaries. This is the fast phase. Changing continuously the reaction rates at the boundaries, there is a point where the relaxation time begins increasing, so that at this point there is a jump in the derivative of the relaxation time with respect to the reaction rates at boundaries. This is the dynamical phase transition and the region where the relaxation time changes 
with reaction rates at boundaries is the slow phase. So the dynamical phase transition studied here, is a discontinuity in the derivative of the relaxation time (from zero to nonzero) with respect to reaction rates at the boundaries.

The scheme of the paper is as follows. In section 2, the model is introduced, and the evolution equation for the spin expectation values is obtained. In section 3 the relaxation of the spin expectation values towards their stationary state is investigated, through a study of the time scales of this relaxation, and a general equation is obtained for these time scales. In section 4, some examples are studied in more detail. It is seen that some of these show two phases, the fast phase and the slow phase. Section 5 is devoted to the concluding remarks, and section 6 is an appendix on the site-link notation and the relaxation time.

\section{One-dimensional Ising model with nonuniform coupling constants}

Consider a one-dimensional lattice with $(L+1)$ sites, labeled from 0 to $L$. At each site $i$, there is a spin variable $s_{i}$, which could be +1 (spin up, $\uparrow$ ), or -1 (spin down, $\downarrow$ ). These spins interact through the Hamiltonian,

$$
\mathcal{H}=\mathcal{H}_{0}^{\prime}+\left(\sum_{\alpha} \mathcal{H}_{\alpha}\right)+\mathcal{H}_{L}^{\prime},
$$

where $\mathcal{H}_{\alpha}$ is the Hamiltonian interaction for the link $\alpha$ :

$$
\mathcal{H}_{\alpha}=-J_{\alpha} s_{\alpha-\mu} s_{\alpha+\mu},
$$

$J_{\alpha}$ is the coupling constant in the link $\alpha$, and

$$
\mu:=\frac{1}{2} .
$$

The link $\alpha$ links the sites $\alpha-\mu$ and $\alpha+\mu$, so that $\alpha \pm \mu$ are integers, and $\alpha$ runs from $\mu$ up to $(L-\mu)$. Throughout this paper, sites are denoted by Latin letters which represent integers, while links are denoted by Greek letters which represent integers plus one half $(\mu)$, so that the link $\alpha$ joins the sites $(\alpha-\mu)$ and $(\alpha+\mu)$, while the site $i$ joins the links $(i-\mu)$ and $(i+\mu) . \mathcal{H}_{0}^{\prime}$ and $\mathcal{H}_{L}^{\prime}$ correspond to interactions at the boundaries. A more extended explanation of the site-link notation is presented in the appendix.

A nonuniform Glauber model gives the dynamics of the Ising model with nonuniform coupling constants $\left(J_{\alpha}\right)$ such that the rate of a spin flip is determined through its interaction with its two neighboring sites and a heat bath at the temperature $T$. Introducing

$$
K_{\alpha}:=\frac{J_{\alpha}}{k_{\mathrm{B}} T},
$$

where $k_{\mathrm{B}}$ is the Boltzmann's constant, it was shown in 26] that assuming nearest neighbor interaction, and that in each step only one spin flips, detailed balance 
gives

$$
\omega\left[\left(S^{\prime}, s_{j}\right) \rightarrow\left(S^{\prime},-s_{j}\right)\right]=\Gamma_{j}\left[1-s_{j} \tanh \left(K_{j-\mu} s_{j-1}+K_{j+\mu} s_{j+1}\right)\right],
$$

where $S^{\prime}$ denotes the configuration of the lattice apart from site $j$, and $\Gamma_{j}$ 's are constants. So that the spin at the site $j$ flips according to the following interactions with the indicated rates.

$$
\begin{array}{lllll}
\uparrow \uparrow \uparrow \rightarrow \uparrow \downarrow \uparrow & \text { and } & \downarrow \downarrow \downarrow \rightarrow \downarrow \uparrow \downarrow & \text { with rate } & 1-\tanh \left(K_{j-\mu}+K_{j+\mu}\right), \\
\uparrow \downarrow \uparrow \rightarrow \uparrow \uparrow \uparrow & \text { and } & \downarrow \uparrow \downarrow \rightarrow \downarrow \downarrow \downarrow & \text { with rate } & 1+\tanh \left(K_{j-\mu}+K_{j+\mu}\right), \\
\uparrow \uparrow \downarrow \rightarrow \uparrow \downarrow \downarrow & \text { and } & \downarrow \downarrow \uparrow \rightarrow \downarrow \uparrow \uparrow & \text { with rate } & 1-\tanh \left(K_{j-\mu}-K_{j+\mu}\right), \\
\downarrow \uparrow \uparrow \rightarrow \downarrow \downarrow \uparrow & \text { and } & \uparrow \downarrow \downarrow \rightarrow \uparrow \uparrow \downarrow & \text { with rate } & 1+\tanh \left(K_{j-\mu}-K_{j+\mu}\right),
\end{array}
$$

where $\Gamma_{j}$ 's have been taken independent of $j$, and set to one by rescaling the time, as in 25, 26. At the boundaries, there are other interactions as well (corresponding to $\mathcal{H}_{0}^{\prime}$ and $\mathcal{H}_{L}^{\prime}$ ). The spin of the zeroth site may flip like

$$
\begin{array}{lll}
\uparrow \downarrow \rightarrow \downarrow & \text { with rate } & g_{1}, \\
\uparrow \uparrow \rightarrow \downarrow \uparrow & \text { with rate } & g_{2}, \\
\downarrow \uparrow \rightarrow \uparrow & \text { with rate } & g_{3}, \\
\downarrow \downarrow \rightarrow \uparrow & \text { with rate } & g_{4},
\end{array}
$$

and the spin of the $L$ 'th site may flip like

$$
\begin{array}{lll}
\downarrow \uparrow \rightarrow \downarrow \downarrow & \text { with rate } & h_{1}, \\
\uparrow \uparrow \rightarrow \uparrow \downarrow & \text { with rate } & h_{2}, \\
\uparrow \downarrow \rightarrow \uparrow & \text { with rate } & h_{3}, \\
\downarrow \downarrow \rightarrow \downarrow \uparrow & \text { with rate } & h_{4} .
\end{array}
$$

It is known that with the rates (6), the time derivatives of the one-point functions in the bulk are expressed in terms of only the one-point functions. To make this true for the boundaries as well, the following relations should hold [17.

$$
\begin{aligned}
g_{1}+g_{4} & =g_{2}+g_{3}, \\
h_{1}+h_{4} & =h_{2}+h_{3} .
\end{aligned}
$$

The first relation, for example, means that the sum of conditional spin flip rates at the zeroth site is independent of the state of the first site. This does not, however, means that the flip rate at the zeroth site is independent of the state of the first site; since, for example, $\left(g_{1}+g_{4}\right)$ itself is not a (conditional) spin flip rate.

Thus the evolution equation for the expectation values of the spins are

$$
\begin{aligned}
\left\langle\dot{s}_{j}\right\rangle= & -2\left\langle s_{j}\right\rangle+\left[\tanh \left(K_{j-\mu}+K_{j+\mu}\right)+\tanh \left(K_{j-\mu}-K_{j+\mu}\right)\right]\left\langle s_{j-1}\right\rangle \\
& +\left[\tanh \left(K_{j-\mu}+K_{j+\mu}\right)-\tanh \left(K_{j-\mu}-K_{j+\mu}\right)\right]\left\langle s_{j+1}\right\rangle, \quad 0<j<L \\
\left\langle\dot{s}_{0}\right\rangle= & \left(g_{3}-g_{1}\right)-\left(g_{2}+g_{3}\right)\left\langle s_{0}\right\rangle+\left(g_{1}-g_{2}\right)\left\langle s_{1}\right\rangle, \\
\left\langle\dot{s}_{L}\right\rangle= & \left(h_{3}-h_{1}\right)-\left(h_{2}+h_{3}\right)\left\langle s_{L}\right\rangle+\left(h_{1}-h_{2}\right)\left\langle s_{L-1}\right\rangle .
\end{aligned}
$$


The static solution $\left(\langle s\rangle_{\mathrm{st}}\right)$ was studied in [26]. Here the dynamics (the relaxation towards the static solution) is addressed.

\section{The relaxation of the system towards equilib- rium}

The homogeneous part of eq. (10) can be written as

$$
\left\langle\dot{s}_{j}\right\rangle=h_{j}^{l}\left\langle s_{l}\right\rangle .
$$

A brief introduction regarding the relaxation time of the system has been contained in the appendix. Denoting an eigenvalue of $h$ by $E$, and the corresponding eigenvector by $x$, it is seen that

$$
\begin{aligned}
E x_{j}= & -2 x_{j}+\left[\tanh \left(K_{j-\mu}+K_{j+\mu}\right)+\tanh \left(K_{j-\mu}-K_{j+\mu}\right)\right] x_{j-1} \\
& +\left[\tanh \left(K_{j-\mu}+K_{j+\mu}\right)-\tanh \left(K_{j-\mu}-K_{j+\mu}\right)\right] x_{j+1}, \quad 0<j<L \\
E x_{0}= & -\left(g_{2}+g_{3}\right) x_{0}+\left(g_{1}-g_{2}\right) x_{1} \\
E x_{L}= & -\left(h_{2}+h_{3}\right) x_{L}+\left(h_{1}-h_{2}\right) x_{L-1}
\end{aligned}
$$

which can be written as

$$
X_{j+\mu}=\tilde{D}_{j} X_{j-\mu},
$$

where

$$
X_{\alpha}:=\left(\begin{array}{c}
x_{\alpha-\mu} \\
x_{\alpha+\mu}
\end{array}\right)
$$

and

$$
\tilde{D}_{j}:=\left(\begin{array}{cc}
0 & 1 \\
-\frac{\sinh \left(2 K_{j-\mu}\right)}{\sinh \left(2 K_{j+h}\right)} & \frac{\cosh \left(2 K_{j-\mu}\right)+\cosh \left(2 K_{j+\mu}\right)}{\sinh \left(2 K_{j+\mu}\right)}\left(1+\frac{E}{2}\right)
\end{array}\right) .
$$

Using the recursion relation (15) repeatedly, one arrives at

$$
X_{\alpha}=\tilde{D}_{\alpha \beta} X_{\beta},
$$

where

$$
\tilde{D}_{\alpha \beta}:=\tilde{D}_{\alpha-\mu} \cdots \tilde{D}_{\beta+\mu}
$$

(13) and (14) are boundary conditions for $X$, and can be rewritten as

$$
\left(\begin{array}{c}
x_{0} \\
x_{1}
\end{array}\right) \propto\left(\begin{array}{c}
g_{1}-g_{2} \\
g_{2}+g_{3}+E
\end{array}\right),
$$


and

$$
\left(\begin{array}{c}
x_{L-1} \\
x_{L}
\end{array}\right) \propto\left(\begin{array}{c}
h_{2}+h_{3}+E \\
h_{1}-h_{2}
\end{array}\right),
$$

or

$$
X_{\mu} \propto\left(\begin{array}{c}
g_{1}-g_{2} \\
g_{2}+g_{3}+E
\end{array}\right),
$$

and

$$
X_{L-\mu} \propto\left(\begin{array}{c}
h_{2}+h_{3}+E \\
h_{1}-h_{2}
\end{array}\right) .
$$

Defining

$$
W:=\left(\begin{array}{ll}
h_{2}-h_{1} & h_{2}+h_{3}+E
\end{array}\right),
$$

it is seen that

$$
W X_{L-\mu}=0 .
$$

Then, using (18) for $\alpha=L-\mu$, and $\beta=\mu$, one arrives at

$$
W \tilde{D}_{L-\mu \mu} V=0 \text {, }
$$

where

$$
V:=\left(\begin{array}{c}
g_{1}-g_{2} \\
g_{2}+g_{3}+E
\end{array}\right) .
$$

(26) is a polynomial equation of order $(L+1)$ for $E$, which has $(L+1)$ roots for $E$. All roots should have nonpositive real parts. The root with largest real part determines the relaxation time of the system. The aim is to investigate the behavior of this root in the thermodynamic limit $(L \rightarrow \infty)$.

\section{Special cases}

1 Uniform coupling constant:

$$
K_{\alpha}=K \text {. }
$$

This is the same kinetic Ising model, the dynamical phase transition of which induced by boundary terms was investigated in [17, using a different method. To fix notation and become familiar with the transfer matrix method, let's use this method for this case. In this case $\tilde{D}_{j}$ is independent of $j$ :

$$
\tilde{D}:=\left(\begin{array}{cc}
0 & 1 \\
-1 & (2+E) \operatorname{coth}(2 K)
\end{array}\right) .
$$

Denoting an eigenvalue of the the matrix $\tilde{D}$ by $z$, it is seen that

$$
E=-2+\left(z+z^{-1}\right) \tanh (2 K) .
$$


So the product of the eigenvalues is one. (26) recasts to

$$
W \tilde{D}^{L-1} V=0 .
$$

$\tilde{D}$ can be written as

$$
\tilde{D}=\frac{1}{z^{-1}-z}\left(\begin{array}{cc}
1 & 1 \\
z & z^{-1}
\end{array}\right)\left(\begin{array}{cc}
z & 0 \\
0 & z^{-1}
\end{array}\right)\left(\begin{array}{cc}
z^{-1} & -1 \\
-z & 1
\end{array}\right),
$$

from which one obtains

$$
\tilde{D}^{L-1}=\frac{1}{z^{-1}-z}\left(\begin{array}{cc}
z^{L-2}-z^{-L+2} & -z^{L-1}+z^{-L+1} \\
z^{L-1}-z^{-L+1} & -z^{L}+z^{-L}
\end{array}\right) .
$$

So (31) becomes

$$
W\left(\begin{array}{cc}
z^{L-2}-z^{-L+2} & -z^{L-1}+z^{-L+1} \\
z^{L-1}-z^{-L+1} & -z^{L}+z^{-L}
\end{array}\right) V=0 .
$$

Substituting $E$, using (30), one arrives from the above equation at a polynomial equation of order $(2 L+4)$ for $z$. Two roots of this equation are \pm 1 , which are not the roots of (31), as (34) has been obtained from (31) by multiplying it by $\left(z^{-1}-z\right)$. The other $(2 L+2)$ roots consist of $(L+1)$ pairs. In each pair the two roots are inverses of each other, and both roots of each pair give the same value for $E$.

The roots of (34) for $z$ are either unimodular or not. For a unimodular solution

$$
z=: e^{\mathrm{i} \theta},
$$

equation (34) becomes

$$
W\left(\begin{array}{cc}
\sin (L-2) \theta & -\sin (L-1) \theta \\
\sin (L-1) \theta & -\sin L \theta
\end{array}\right) V=0,
$$

which can be written like

$$
F(\theta)=0 .
$$

It is seen that in the thermodynamic limit, the entries of the matrix appearing in the left-hand side of (36) change sign when the value of $\theta$ is changed by $(\pi / L)$, while $V$ and $W$ do not change. So the sign of $F(\theta)$ is opposite of the sign of $F[\theta+(\pi / L)]$, meaning that for any value of $\theta_{0}$ there is at least one root for $\theta$ between $\theta_{0}$ and $\left[\theta_{0}+(\pi / L)\right]$. So, in the thermodynamic limit all of unimodular $z$ 's are roots of (34). These correspond to real values of $E$, with the maximum

$$
E_{0}=-2+2 \tanh (2|K|),
$$


which corresponds to the relaxation time

$$
\tau_{0}=\frac{1}{2-2 \tanh (2|K|)} .
$$

If there are no nonunimodular solutions for $z$, this is the relaxation time of the system, which does not depend on the reaction rates at the boundaries. Let's call this the fast phase. If, however, there is a value for $E$ the real part of which is larger than the right-hand side of (38), then the relaxation time is larger than $\tau_{0}$ and, as it will be seen, does depend on the boundaries. This is called the slow phase.

If there is a nonunimodualr root for $z$, then there is a root $z$ with modulus larger than one. Then, in the thermodynamic limit (34) becomes (for this root)

$$
W\left(\begin{array}{cc}
z^{-1} & -1 \\
& -z
\end{array}\right) V=0
$$

or

$$
\left[\left(h_{2}-h_{1}\right) z^{-1}+h_{2}+h_{3}+E\right]\left[\left(g_{2}-g_{1}\right) z^{-1}+g_{2}+g_{3}+E\right]=0 .
$$

Using (30), this equation turns out to be

$$
\begin{aligned}
& \left\{\left[h_{2}-h_{1}+\tanh (2 K)\right] z^{-1}+[\tanh (2 K)] z+h_{2}+h_{3}-2\right\} \\
\times & \left\{\left[g_{2}-g_{1}+\tanh (2 K)\right] z^{-1}+[\tanh (2 K)] z+g_{2}+g_{3}-2\right\}=0,
\end{aligned}
$$

which is exactly the same as equation (27) in [17. So the rest of discussion is exactly similar to that of [17. The root obtained from (42) does depend on the reaction rates at the boundaries. Such a root corresponds to a relaxation time larger than $\tau_{0}$, iff

$$
\left[\operatorname{Re}\left(z+z^{-1}\right)\right][\operatorname{sgn}(K)]>2 .
$$

This gives the following coexistence surface for the two phases

$$
g_{2}+g_{3}+[\operatorname{sgn}(K)]\left(g_{2}-g_{1}\right)-2[1-\tanh (2|K|)]=0,
$$

or

$$
\begin{aligned}
& {\left[4 \tanh (2|K|)+2-g_{2}-g_{3}\right]\left[\tanh (2|K|)-g_{1}+g_{2}\right] } \\
+ & {[\tanh (2|K|)]\left(g_{2}+g_{3}-2\right)=0, }
\end{aligned}
$$

and similar solutions with $h_{i}$ 's substituting $g_{i}$ 's.

In summary, for some values of reaction rates at the boundaries the relaxation time is $\tau_{0}$, which is independent of the reaction rates at the boundaries. At a certain point, however, the relaxation time starts increasing from $\tau_{0}$ and being dependent on the reaction rates at the boundaries. The 
dynamical phase transition is this discontinuous change of the derivative of the relaxation time towards the stationary configuration.

As examples for this transition, let's consider two cases. Regarding the coexistence curve (44), it is seen that for

$$
\begin{aligned}
& g_{1}=g_{2}, \\
& g_{4}=g_{3},
\end{aligned}
$$

one has

$$
\left\{\begin{array}{ll}
g_{2}+g_{3}<2[1-\tanh (2|K|)], & \text { slow phase } \\
g_{2}+g_{3}>2[1-\tanh (2|K|)], & \text { fast phase }
\end{array} .\right.
$$

Regarding the coexistence curve (45), it is seen that for

$$
\begin{aligned}
g_{1} & =g_{3}, \\
g_{4} & =g_{2}, \\
g_{2}+g_{3} & =2,
\end{aligned}
$$

one has

$$
\begin{cases}g_{3}-g_{2}<\tanh (2|K|), & \text { slow phase } \\ g_{3}-g_{2}>\tanh (2|K|), & \text { fast phase }\end{cases}
$$

$\mathbf{2} K_{\alpha}= \begin{cases}K^{\prime}, & \alpha<L^{\prime} \\ K, & \alpha>L^{\prime}\end{cases}$

It has been assumed that in the thermodynamic limit both $L^{\prime}$ and $\left(L-L^{\prime}\right)$ tend to infinity. Also, without loss of generality it can be assumed that $|K|>\left|K^{\prime}\right|$. This is a lattice consisting of two parts, on each part the coupling constant is uniform. Equation (26) becomes

$$
W \tilde{D}^{L-L^{\prime}-1} \tilde{D}_{L^{\prime}} \tilde{D}^{L^{\prime}-1} V=0
$$

where $\tilde{D}$ is defined through (29), $\tilde{D}^{\prime}$ is similar to $\tilde{D}$ but with $K$ replaced by $K^{\prime}$, and

$$
\tilde{D}_{L^{\prime}}:=\left(\begin{array}{cc}
0 & 1 \\
-\frac{\sinh \left(2 K^{\prime}\right)}{\sinh (2 K)} & \frac{\cosh \left(2 K^{\prime}\right)+\cosh (2 K)}{\sinh (2 K)}\left(1+\frac{E}{2}\right)
\end{array}\right) .
$$

Denoting an eigenvalue of $\tilde{D}\left(\tilde{D}^{\prime}\right)$ by $z\left(z^{\prime}\right)$, it is seen that (30) holds and

$$
E=-2+\left(z^{\prime}+z^{\prime-1}\right) \tanh \left(2 K^{\prime}\right)
$$

Equations (50), (30), and (52) are used to obtain the values of $z$ and $z^{\prime}$. First assume that $z$ is unimodular. Then $E$ is real and from (52) it is seen that $z^{\prime}$ is either unimodular or real. If $z^{\prime}$ is unimodular too, then the 
possible values for $z$ are not all of the points of the unit circle. Defining $\theta$ by (35), it is seen that those values of $\theta$ which correspond to unimodular values for $z^{\prime}$ are

$$
\cos ^{-1}\left[\frac{\tanh \left(2\left|K^{\prime}\right|\right)}{\tanh (2|K|)}\right] \leq|\theta| \leq \pi-\cos ^{-1}\left[\frac{\tanh \left(2\left|K^{\prime}\right|\right)}{\tanh (2|K|)}\right] .
$$

For each value of $\theta$ out of this region, there are two real values for $z^{\prime}$ which are inverses of each other. For that value of $z^{\prime}$ the modulus of which is greater than one, one arrives from (50) at

$$
W\left(\begin{array}{cc}
\sin \left(L-L^{\prime}-2\right) \theta & -\sin \left(L-L^{\prime}-1\right) \theta \\
\sin \left(L-L^{\prime}-1\right) \theta & -\sin \left(L-L^{\prime}\right) \theta
\end{array}\right) \tilde{D}_{L^{\prime}}\left(\begin{array}{cc}
z^{\prime-1} & -1 \\
1 & -z^{\prime}
\end{array}\right) V=0,
$$

again showing that if $\left(L-L^{\prime}\right)$ tends to infinity, all of the (remaining) values of $\theta$ are roots of this equation. So one piece of the solutions for $E$ corresponds to the full unit circle for $z$ and the full unit circle plus two real segments for $z^{\prime}$. The relaxation time corresponding to these eigenvalues is $\tau_{0}$ from (39). It is seen that it is the larger coupling which determines the relaxation time (provided the part of the lattice which corresponds to this coupling becomes infinite). If all of the solutions for $z$ are unimodular, $\tau_{0}$ is the relaxation time of the system and the system is in the fast phase.

The system would be in the slow phase (where the relaxation time is larger than $\tau_{0}$ and does depend on the rates at the boundaries) iff there exists a solution for $z$ for which (43) holds. In this case, one arrives at

$$
W\left(\begin{array}{cc}
z^{-1} & -1 \\
1 & -z
\end{array}\right) \tilde{D}_{L^{\prime}}\left(\begin{array}{cc}
z^{\prime-1} & -1 \\
1 & -z^{\prime}
\end{array}\right) V=0
$$

where $z$ and $z^{\prime}$ have are solutions which have moduli larger than one. One then arrives at

$$
\begin{aligned}
& \left\{\left[h_{2}-h_{1}+\tanh (2 K)\right] z^{-1}+[\tanh (2 K)] z+h_{2}+h_{3}-2\right\} \\
\times & {\left[z \sinh (2 K)+z^{\prime} \sinh \left(2 K^{\prime}\right)\right] } \\
\times & \left\{\left[g_{2}-g_{1}+\tanh \left(2 K^{\prime}\right)\right] z^{\prime-1}+\left[\tanh \left(2 K^{\prime}\right)\right] z^{\prime}+g_{2}+g_{3}-2\right\}=0 .
\end{aligned}
$$

From (30) and (52), it is seen that the real parts of $[z \tanh (2 K)]$ and $\left[z^{\prime} \tanh \left(2 K^{\prime}\right)\right]$, and hence the real parts of $[z \sinh (2 K)]$ and $\left[z^{\prime} \sinh \left(2 K^{\prime}\right)\right]$ have the same sign, or both are zero. So the second factor in the left hand side of (56) does not vanish unless $z$ and $z^{\prime}$ are $( \pm \mathrm{i})$. But these values of $z$ and $z^{\prime}$ are unimodular hence not acceptable, as the roots of (56). So in order that (56) is satisfied, either the first or the third factor in the left hand side of (56) should vanish. This is similar to (42), with the couplings corresponding to each part of the lattice instead of the uniform coupling. One then arrives at (44) and (45) with $K$ replaced by $K^{\prime}$, or 
similar expressions with $K^{\prime}$ and $g_{i}$ 's replaced by $K$ and $h_{i}$ 's respectively, as the coexistence surface. Of course from the possible roots of (56), that root is chosen which corresponds to the larger relaxation time (or larger real part for $E$ ), and one should also check that this root satisfies (43).

To summarize, in the fast phase the relaxation time is determined by the coupling with larger modulus. In the slow phase the two ends of the lattice behave independently, the relaxation time being determined by the largest.

$3 K_{\alpha}= \begin{cases}K^{\prime}, & \alpha<L^{\prime} \\ K_{\alpha}, & L^{\prime}<\alpha<L-L^{\prime \prime} \\ K^{\prime \prime}, & L-L^{\prime \prime}<\alpha\end{cases}$

It has been assumed that in the thermodynamic limit both $L^{\prime}$ and $L^{\prime \prime}$ tend to infinity, while $\left(L-L^{\prime}-L^{\prime \prime}\right)$ remains finite. This is a lattice with a finite part inside which, where outside that part the coupling is uniform. Equation (26) becomes

$$
W \tilde{D}^{\prime \prime L^{\prime \prime}-1} \tilde{D}_{L-L^{\prime \prime}+\mu L^{\prime}-\mu} \tilde{D}^{L^{\prime}-1} V=0,
$$

where $\tilde{D}^{\prime}$ and $\tilde{D}^{\prime \prime}$ are defined through (29), but with $K$ replaced by $K^{\prime}$ and $K^{\prime \prime}$ respectively. Similar to the previous example, one defines $z^{\prime}$ and $z^{\prime \prime}$ similar to (30) and (52). Again similar to the previous example, there is a relaxation time corresponding to the fast phase, where from $z^{\prime}$ and $z^{\prime \prime}$ at least one is unimodular, which is obtained from (39), with $|K|$ being the larger between $\left|K^{\prime}\right|$ and $\left|K^{\prime \prime}\right|$. In order that the system be in the slow phase, there should be a solution where both $z^{\prime}$ and $z^{\prime \prime}$ are nonunimodular. As for any value of $E$, the product of the eigenvalues of $\tilde{D}_{i}$ is one, one can take $z^{\prime}$ and $z^{\prime \prime}$ so that both have moduli larger than one. One then arrives at

$$
W\left(\begin{array}{cc}
z^{\prime \prime-1} & -1 \\
1 & -z^{\prime \prime}
\end{array}\right) \tilde{D}_{L-L^{\prime \prime}+\mu L^{\prime}-\mu}\left(\begin{array}{cc}
z^{\prime-1} & -1 \\
1 & -z^{\prime}
\end{array}\right) V=0
$$

or

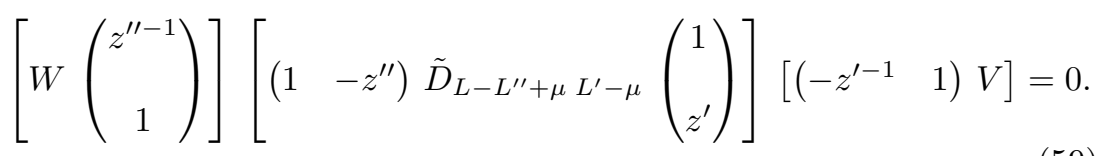

Putting the first or the third factor equal to zero, a result similar to that of the previous example is obtained. But there may be another solution which is obtained by letting the second factor vanish:

$$
\left(\begin{array}{ll}
1 & -z^{\prime \prime}
\end{array}\right) \tilde{D}_{L-L^{\prime \prime}+\mu L^{\prime}-\mu}\left(\begin{array}{c}
z^{-1} \\
1
\end{array}\right)=0
$$


This new solution (if it exists) can change the relaxation time. If the relaxation time corresponding to the solution to (60) is larger, then this relaxation time is the relaxation time in the slow phase. So, the introduction a finite part in the lattice can change the relaxation time and hence the coexistence surface. In fact, qualitatively this finite part plays the role of new boundaries introduced in the lattice.

4 Alternating Coupling constant $K_{\alpha}=(-1)^{\alpha-\mu} K$

Defining

$$
\begin{aligned}
\tilde{D}_{\text {o }} & :=\left(\begin{array}{cc}
0 & 1 \\
1 & -(2+E) \operatorname{coth}(2 K)
\end{array}\right) \\
\tilde{D}_{\mathrm{e}} & :=\left(\begin{array}{cc}
0 & 1 \\
1 & (2+E) \operatorname{coth}(2 K)
\end{array}\right),
\end{aligned}
$$

it is seen that (26) becomes

$$
W \tilde{D}_{\mathrm{o}}^{1-r}\left(\tilde{D}_{\mathrm{e}} \tilde{D}_{\mathrm{o}}\right)^{(L-2+r) / 2} V=0
$$

where $r$ is 0 (1) when $L$ is even (odd). Defining $z$ through

$$
E=-2+\left(z+z^{-1}\right) \tanh (2 K)
$$

it is then seen that

$$
\tilde{D}_{\mathrm{e}} \tilde{D}_{\mathrm{o}}=\left(\begin{array}{cc}
1 & -\left(z+z^{-1}\right) \\
\left(z+z^{-1}\right) & 1-\left(z+z^{-1}\right)^{2}
\end{array}\right),
$$

showing that the eigenvalues of $\left(\tilde{D}_{\mathrm{e}} \tilde{D}_{\mathrm{o}}\right)$ are $\left(-z^{2}\right)$ and $\left(-z^{-2}\right)$. So,

$$
\left(\tilde{D}_{\mathrm{e}} \tilde{D}_{\mathrm{o}}\right)^{q}=\frac{(-1)^{q}}{z^{-1}-z}\left(\begin{array}{cc}
1 & 1 \\
z & z^{-1}
\end{array}\right)\left(\begin{array}{cc}
z^{2 q} & 0 \\
0 & z^{-2 q}
\end{array}\right)\left(\begin{array}{cc}
z^{-1} & -1 \\
-z & 1
\end{array}\right) \text {. }
$$

Again (63) shows that $\tau_{0}$ is the relaxation time of the system (the system is in the fast phase), if all of the values of $z$ are unimodular. To have a larger relaxation time (the system be in the slow phase), there should be a value of $z$ with modulus greater than 1 . In fact, there should be a value of $z$ satisfying (43). In that case, in the thermodynamic limit $(q \rightarrow \infty)$ one arrives from (62) to

$$
\left[W \tilde{D}_{\circ}^{1-r}\left(\begin{array}{c}
z^{-1} \\
1
\end{array}\right)\right]\left[\left(\begin{array}{ll}
z^{-1} & -1) V
\end{array}\right]=0,\right.
$$


or

$$
\left[W\left(\begin{array}{c}
(-1)^{1-r} z^{-1} \\
1
\end{array}\right)\right]\left[\begin{array}{ll}
z^{-1} & -1) V
\end{array}\right]=0 .
$$

It is seen that for $r=1$ (odd $L$ ), this is exactly (42). For $r=0$ (even $L$ ), the second factor in the left-hand side of (66) is the same as the second factor in (42), while the first factor is the first factor in (42) with $z$ and $K$ replaced by $(-z)$ and $(-K)$, respectively. So the coexistence surfaces (44) and (45) are recovered here as well, while the coexistence surfaces corresponding to $h_{i}$ 's are the same as those obtained for the uniform lattice if $L$ is odd, and the same with $K$ replaced by $(-K)$ if $L$ is even.

One could also arrive at this result by making a correspondence between this case (alternating coupling constants) and case 1 (uniform coupling constants). The analogy goes as the following.

$$
\begin{aligned}
& \tilde{x}_{j}:=(-1)^{[j / 2]} x_{j}, \\
& \tilde{K}_{\alpha}:=(-1)^{\alpha-\mu} K_{\alpha}, \\
&\left(\tilde{g}_{1}, \tilde{g}_{2}, \tilde{g}_{3}, \tilde{g}_{4}\right):=\left(g_{1}, g_{2}, g_{3}, g_{4}\right), \\
&\left(\tilde{h}_{1}, \tilde{h}_{2}, \tilde{h}_{3}, \tilde{h}_{4}\right): \\
&:\left\{\begin{array}{ll}
\left(h_{1}, h_{2}, h_{3}, h_{4}\right), & \text { for odd } L \\
\left(h_{2}, h_{1}, h_{4}, h_{3}\right), & \text { for even } L
\end{array},\right.
\end{aligned}
$$

where $[y]$ means the largest integer not greater than $y$. It is seen that in this way, if the untilded varibles and constants satisfy (10) till (12), the tilded varibles and constants satisfy (10) till (12) as well, and if $K_{\alpha}$ is alternating, $\tilde{K}_{\alpha}$ is obviously uniform. The meaning of this correspondence between $\tilde{x}_{j}$ 's and $x_{j}$ 's is that, for example, $x_{0}$ and $x_{1}$ are kept fixed, while $x_{2}$ and $x_{3}$ have been changed to $\left(-x_{2}\right)$ and $\left(-x_{3}\right)$, respectively, and $K_{1 / 2}$ and $K_{5 / 2}$ have been kept fixed, while $K_{3 / 2}$ has been changed to $\left(-K_{3 / 2}\right)$. Obviously the expressions $\left(K_{1 / 2} x_{0} x_{1}\right),\left(K_{3 / 2} x_{1} x_{2}\right),\left(K_{5 / 2} x_{2} x_{3}\right)$ have all remained intact, while the coupling is now uniform, if it has been alternating. If $L$ is odd, no change in the boundary rates is required and the coexistence curves would be exactly the same as those found in the case of uniform coupling. If $L$ is even, that part of the coexistence curve which arises from the $g$ 's is still intact, as $g$ 's have remained intact. But the part due to $h$ 's is changed. And the change comes from the fact that $\left(h_{2}-h_{1}\right)$ has been changed to $\left(h_{1}-h_{2}\right)$, while $\left(h_{2}+h_{3}\right)$ has remained intact. One can see that in the equations of the coexistence curves involving $h$ 's (44) and (45) but with $g$ 's replaced with $h$ 's), this change is equivalent to a change of $z$ to $(-z)$ and $K$ to $(-K)$.

\section{Concluding remarks}

A one dimensional Ising model with nonuniform coupling constants on a lattice with boundaries was studied. Detailed balance was used to obtain the evolu- 
tion of this model. The relaxation of the resulted system towards its stationary solution was studied. A general formulation was obtained to determine the relaxation times and possible dynamical phase transitions, where the system moves from the fast phase (with the relaxation time independent of boundaries) to the slow phase (with the relaxation time depending on boundaries). Some special examples were studied in more detail, including cases where the lattice consists of two infinite homogeneous parts and one middle (possibly nonhomogeneous part), where it was shown that this middle part can induce further dynamical phase transitions, behaving like an effective boundary.

Part of the results obtained here is similar to those corresponding to the case of uniform coupling constants. The similarity is that the system is in the fast phase when the boundary couplings are high enough so that it is the bulk reaction rates that determine the relaxation, and goes to the slow phase when the boundary reactions are less than some critical value. Another similarity is that each boundary behaves essentially independent to the other boundary. When the system consists of large parts which are essentially uniform, the new feature in the relaxation is that the system behaves as though it is consists of independent systems each having two boundaries. So for such systems new coexistence curves arise. There are of course cases where no large uniform blocks are present. Then the only similarity with the uniform case is that increasing the boundary rates makes them unimportant in the relaxation time.

\section{Appendix}

\section{1 notation for a lattice}

A directional lattice is a collection of sites and directional links, with a relation between links and sites. A link $\mathbf{l}$ has a negative boundary (denoted by $\partial^{-} \mathbf{l}$ ), and a positive boundary (denoted by $\partial^{+} \mathbf{l}$ ). The relation of the site $i$ to the link $\mathbf{l}$ is that either $i$ is $\partial^{-} \mathbf{l}$, or $i$ is $\partial^{+} \mathbf{l}$, or $i$ is not in $\mathbf{l}$. To each site $i$ there corresponds a set of outgoing links $S^{+}(i)$, and a set of incoming links $S^{-}(i)$ :

$$
\begin{aligned}
& S^{+}(i):=\left\{\mathbf{l} \mid \partial^{-} \mathbf{l}=i\right\}, \\
& S^{-}(i):=\left\{\mathbf{l} \mid \partial^{+} \mathbf{l}=i\right\} .
\end{aligned}
$$

In a one dimensional (connected) lattice these relations become simple. The lattice sites can be denoted by integers from a subset $Q$ of the integers, with the property that if an integer is between two members of $Q$, it is a member of $Q$ itself. $Q$ can be bounded from either above or below. If it is bounded from above, then the lattice has a positive boundary, denoted by $\partial^{+} Q$, which is the largest member of $Q$. Similarly the smallest member of $Q$ (if it exists) is the negative boundary of $Q$ which is denoted by $\partial^{-} Q . S^{+}(i)$ consists of exactly one link, unless $i$ is $\partial^{+} Q$, in which case $S^{+}(i)$ is empty. Similarly, $S^{-}(i)$ consists of exactly one link, unless $i$ is $\partial^{-} Q$, in which case $S^{-}(i)$ is empty. So one can denote the outgoing and incoming links corresponding to the site $i$, simply 
through

$$
i \pm \mu:=S^{ \pm}(i)
$$

from which one also has

$$
\partial^{ \pm} \alpha=\alpha \pm \mu .
$$

If the lattice is closed and has $L$ sites, one can still use the above notation, provided an equivalence between $b$ and $(b+L)$ is assumed, where $b$ can be a site (an integer) or a link (an integer plus half).

\section{2 the relaxation time}

Denoting the deviation from the static configuration of the system by $x$, it is seen that $x$ satisfies

$$
\dot{y}=h y,
$$

which is nothing but the closed form of (11). To solve this, one expresses $y$ as a linear combination of the (generalized) eigenvectors of $h$ :

$$
y(t)=\sum_{\lambda} c_{\lambda}(t) x_{\lambda},
$$

where $x_{\lambda}$ is the (generalized) eigenvector of $h$ corresponding to the eigenvalue $E_{\lambda}$. If all of the generalized eigenvectors are eigenvectors, evolution equations for $c_{\lambda}$ 's decouple as

$$
\dot{c}_{\lambda}=E_{\lambda} c_{\lambda}
$$

resulting in

$$
c_{\lambda}(t)=c_{\lambda}(0) \exp \left(E_{\lambda} t\right) .
$$

So one arrives at

$$
y(t)=\sum_{\lambda} c_{\lambda}(0) \exp \left(E_{\lambda} t\right) x_{\lambda} .
$$

Initial condition determines $c_{\lambda}(0)$ 's. It is seen that for large times the leading term of the right hand side of (76) is that term which corresponds to the largest real part of $E_{\lambda}$, unless the initial condition is fine tuned so that $c_{\lambda}(0)$ vanishes for that $\lambda$. So the relaxation time is

$$
\tau=-\frac{1}{\max \left[\operatorname{Re}\left(E_{\lambda}\right)\right]} .
$$

In the thermodynamic limit, it may happen that the maximum of $\operatorname{Re}\left(E_{\lambda}\right)$ tends to zero, so that the relaxation time goes to infinity, meaning that the relaxation is no longer exponential but say power law. But it may also happen that this is not the case, and the maximum of $\operatorname{Re}\left(E_{\lambda}\right)$ tends to a negative number, in which case the relaxation time remains finite even in the thermodynamic limit.

Acknowledgement: This work was partially supported by the research council of the Alzahra University. 


\section{References}

[1] R. J. Glauber; J. Math. Phys. 4 (1963) 294.

[2] K. Kawasaki; Phys. Rev. 145 (1966) 224.

[3] J. G. Amar \& F. Family; Phys. Rev. A41 (1990) 3258.

[4] T. Vojta; Phys. Rev E55 (1997) 5157.

[5] R. B. Stinchcombe, J. E. Santos, \& M. D. Grynberg; J. Phys. A31 (1998) 541.

[6] C. Godrèche \& J. M. Luck; J. Phys. A33 (2000) 1151.

[7] E. Lippiello \& M. Zannetti; Phys. Rev. E61 (2000) 3369.

[8] M. Droz, Z. Rácz, \& J. Schmidt; Phys. Rev. A39 (1989) 2141.

[9] B. C. S. Grandi \& W. Figueiredo; Phys. Rev. E53 (1996) 5484.

[10] S. Artz \& S. Trimper; Int. J. Mod. Phys. B12 (1998) 2385.

[11] M. Khorrami \& A. Aghamohammadi; Phys. Rev. E63 (2001) 042102.

[12] B. Schmittmann \& F. Schmüser; Phys. Rev. E66 (2002) 046130.

[13] B. Schmittmann \& F. Schmüser; J. Phys. A35 (2002) 2569.

[14] M. Mobilia, R. K. P. Zia, \& B. Schmittmann; J. Phys. A37 (2004) L407.

[15] C. Chatelain; J. Phys. A36 (2003) 10739.

[16] M. Droz, J. Kamphorst Leal da Silva, A. Malaspinas, \& A. L. Stella; J. Phys. A20 (1987) L387.

[17] M. Khorrami \& A. Aghamohammadi; Phys. Rev. E65 (2002) 056129.

[18] M. Henkel \& G. Schütz; Physica A206 (1994) 187.

[19] M. J. E. Richardson \& Y. Kafri; Phys. Rev. E59 (1999) R4725.

[20] A. Aghamohammadi \& M. Khorrami; J. Phys. A34 (2001) 7431.

[21] A. Shariati, A. Aghamohammadi, \& M. Khorrami; Phys. Rev. E64 (2001) 066102 .

[22] S. N. Majumdar, D. S. Dean, \& P. Grassberger Phys. Rev. Lett. 86 (2001) 2301.

[23] N. Menyhard \& G. Odor; Phys. Rev. E76 (2007) 021103.

[24] B. Deviren, M. Keskin, O. Canko; J. Magn. Magn. Mater. 321 (2009) 458

[25] J. C. Angles d'Auriac \& R. Rammal; J. Phys. A21 (1988) 763.

[26] M. Khorrami \& A. Aghamohammadi; arXiv: 0811.2283. 